\title{
Development of an experimental model to evaluate bupivacaine induced \\ ventricular arrhythmias in a porcine experimental model. Preliminary evaluation of intralipid
}

Authors: López-Menchaca R, Callejo D, Sevilla R, Salas J, Quintela O, Zaballos M Hospital Universitario Gregorio Marañón. Universidad Complutense. Madrid, Spain.

\section{Background and Goal of Study}

Unintentional intravenous administration of bupivacaine might produce life-threatening arrhythmias. Surprisingly, despite the fact that several clinical reports and animal studies have described the occurrence of lethal ventricular arrhythmias associated with bupivacaine intoxication, there is limited and confusing information regarding essential characteristics of these arrhythmias. Furthermore, no arrhythmias were provoked in some reports of bupivacaine intoxication after ventricular pacing (1) Our aim was to develop a reproducible model to study paced induced ventricular arrhythmias in the context of bupivacaine intoxication, and evaluate if intralipid administration protect of these arrhythmias

\section{Material and methods}

Six Large-White pigs were premedicated with ketamine and anesthetized with intravenous sodium thiopental $5 \mathrm{mg}-\mathrm{kg}-1$. The anesthetic maintenance was performed with sevoflurane $1 \mathrm{MAC}$ (2.6\%). Femoral artery and vein were canalized for invasive monitoring, analytical blood gas samples and bupivacaine levels determinations. Two quadripolar catheters were used for stimulation and intracardiac recordings and advanced to the high right atrium and to the right ventricular apex. After instrumentation a bupivacaine bolus of $4 \mathrm{mg} . \mathrm{kg}-1$ was administered followed by a continuous infusion of $100 \mu \mathrm{g} . \mathrm{kg}-1 . \mathrm{min}-1$. A modified programmed ventricular stimulation protocol was performed (at baseline and after 15 min of bupivacaine perfusion) (2) The ventricle was paced at maximal current strength (at three basic cycle lengths (350, 400 and $600 \mathrm{~ms}$ ). After an 8 beat pacing train, programmed stimulation was initiated with coupling intervals of $290,280,270$, and 260 ms for the first through fourth extrastimuli. In two additional animals an IL infusion was administrated three minutes after bupivacaine bolus dose.

\section{Results}

At baseline, no animals except one, developed ventricular arrhythmias after the ventricular stimulation protocol, however sustained ventricular tachydysrhythmias occurred in $87,5 \%$ of the animals with the administration of bupivacaine.(Fig 1) The administration of intralipid did not prevent the development of ventricular arrhythmias

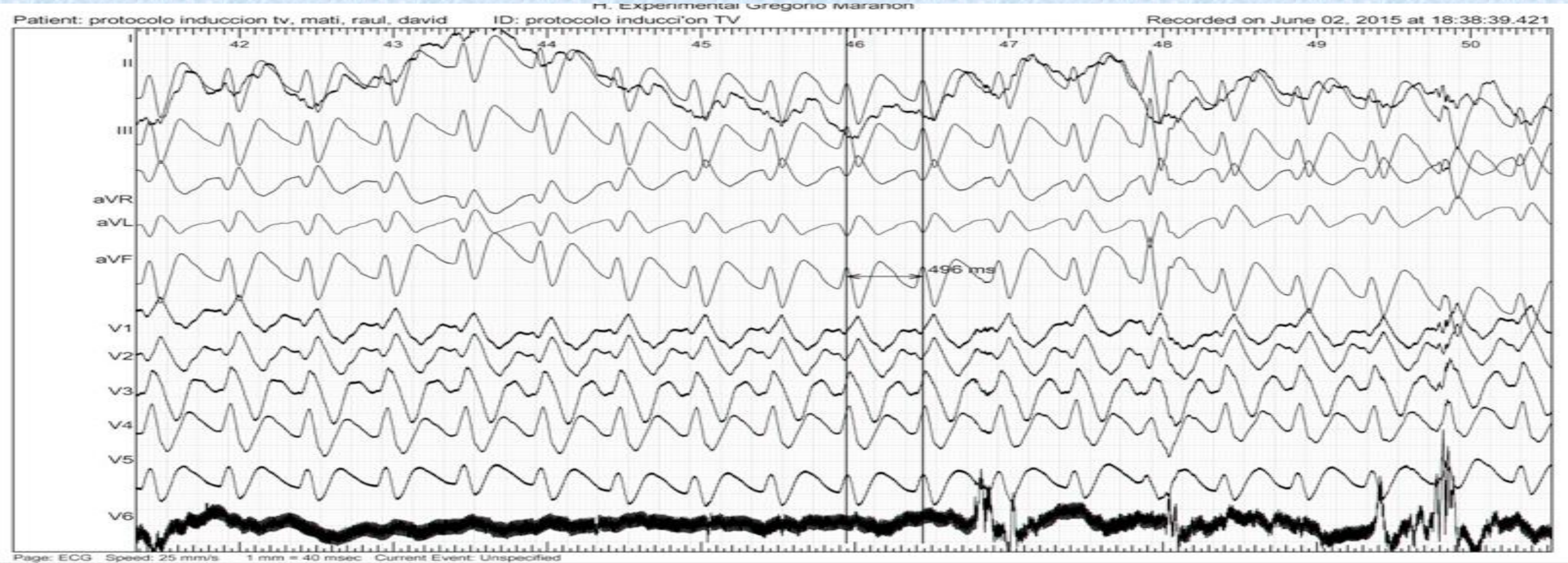

Figure 1. An example of a polymorphic ventricular tachycardia elicited after stimulation protocol during bupivacaine administration.

\section{Conclusion}

We have shown that this experimental model is useful to evaluate and investigate bupivacaine-induced ventricular arrhythmias. Our preliminary data show that intralipid was not effective to prevent paced-induced ventricular arrhythmias in the context of a porcine experimental model of bupivacaine intoxication.

This study is supported by a FEDER and FIS grant

\section{References:}

